\title{
Microinvasive Glaucoma Surgery-A New Chapter in Glaucoma Management
}

\author{
Mina B Pantcheva \\ University of Colorado Health Eye Center, Aurora, Colorado, US
}

DOl:http://doi.org/10.17925/USOR.2016.09.01.30

\begin{abstract}
The continuous search for techniques and devices that lower the intraocular pressure without the complications associated with our gold standard trabeculectomy procedure has led to some promising developments in glaucoma surgery. The newly emerging technologies place improved patient safety as a priority. This article offers a brief overview of the current status and future innovations of the microinvasive glaucoma surgery (MIGS) and its role in our glaucoma practice.
\end{abstract}

\section{Keywords}

microinvasive, ab interno, glaucoma, surgery, cataract, open-angle glaucoma

Disclosure: Mina B Pantcheva has no conflicts of interest to declare. No funding was received in the publication of this article. This article is a short opinion piece and has not been submitted to external peer reviewers.

Open Access: This article is published under the Creative Commons Attribution Noncommercial License, which permits any noncommercial use, distribution, adaptation, and reproduction provided the original author(s) and source are given appropriate credit.

Received: February 21, 2016 Published Online: 17 March, 2016 Citation: US Ophthalmic Review, 2016;9(1):30-1

Correspondence: Mina B Pantcheva, University of Colorado Health Eye Center, 1675 Aurora Court, Aurora, Co 80045, US. E: mina.pantcheva@ucdenver.edu

The development of innovative microinvasive glaucoma surgery (MIGS) procedures has given us an opportunity to fill a long-existing niche in the glaucoma management. Traditionally, the treatment for mild to moderate glaucoma includes noninvasive and relatively safe topical medications and laser trabeculoplasty. MIGS offers new options for patients with mild to moderate open-angle glaucoma (OAG) who have failed medical or laser therapy but whose disease is not so advanced to warrant filtering surgeries, which pose a risk of vision-threatening complications from the surgery itself. This article offers a glimpse at the current and latest developments of MIGS.

MIGS procedures involve an ab interno, microincisional, conjunctivasparing approach with minimal tissue trauma and disruption of normal anatomy and physiology. ${ }^{1}$ The devices used for implantation exhibit a high level of biocompatibility. MIGS achieves moderate to high intraocular pressure (IOP) lowering efficacy, resulting in IOP in the mid-teens. The surgical techniques have a very good safety profile and allow for rapid recovery by the patient. All MIGS procedures are performed under gonioscopic view through a small side port incision. The ab interno techniques include removing trabecular meshwork tissue or implanting a shunt device.

\section{Trabectome}

Ab interno trabeculotomy by the Trabectome ${ }^{\circledR}$ device (NeoMedix, Tustin, USA) uses a high frequency electrocautery to remove a strip of trabecular meshwork and the inner wall of Schlemm's canal (SC). ${ }^{2}$ The rationale for this angle surgery is the removal of the area of greatest resistance to aqueous outflow while achieving some retraction of the incision edges by heat effect and consequently reducing potential scarring. The device consists of a disposable hand piece, controlled via a 3-stage Foot Pedal Control that initiates irrigation, aspiration and electrocautery in sequence. No randomized controlled trials have been performed to date. Several studies have shown that the Trabectome alone and combined with phacoemulsification results in IOP levels ranging from 13.5 to $17.9 \mathrm{mmHg}$ with greater reduction in patients with higher preoperative IOP and medication reduction from $21 \%$ to $38 \% .^{3-6}$ Jea and coworkers compared the safety and efficacy of the Trabectome to trabeculectomy with mitomycin $\mathrm{C}$ and concluded that the success rate of Trabectome was significantly lower. However, its excellent safety profile makes it a viable option for patients in which the risks of trabeculectomy are of particular concern, and patients in early disease stage, which typically involves more modest IOP goals.?

\section{iStent}

The iStent ${ }^{\circledR}$ trabecular micro-bypass stent (Glaukos, Laguna Hills, CA, USA) is a heparin-coated, non-ferromagnetic titanium device with a snorkel shape to facilitate implantation into SC and three retention arches in its outer surface to ensure secure placement. It is approximately $1 \times 0.3 \mathrm{~mm}$ in size. It is placed using a single-use sterile inserter and directly connects the anterior chamber with SC. A systematic review and meta-analysis has shown that the istent as a solo procedure without concurrent cataract surgery does lower IOP and reduces the dependency on glaucoma medications for up to 18 months post surgery. ${ }^{8}$ Although the data are limited, it suggests that the IOP decrease correlates with the number of istents implanted. A 22\% IOP reduction from baseline occurred at 18 months after one istent implant, 30\% at 6 months after implantations 
of two istents, and $41 \%$ at 6 months after implantation of three iStents. The authors found a notable lack of published research on rates of early and late post-operative complications. The most common complications were related to istent malposition or obstruction.

\section{CyPass Micro-Stent}

The CyPass $^{\circledR}$ Micro-Stent (Transcend Medical Inc, Menlo Park, CA) is currently for investigational use in the United States. The rationale behind this polyimide, supraciliary device is to create a controlled cyclodialysis with stented outflow to the suprachoroidal space. This space is an attractive target due to its large surface area and negative pressure gradient, providing driving force for the aqueous outflow. The device is a $6.35 \mathrm{~mm}$ long tube with $300 \mu \mathrm{m}$ lumen. It is inserted on a small guidewire with a special tip that separates the iris from the scleral spur allowing for the device to be inserted into the created cleft. Once the tube is in place, openings along its length allow aqueous to flow out. Hoeh et al. reported the one-year outcomes of the CyPass Clinical Experience (CyCLE) study. ${ }^{9}$ All subjects had OAG and underwent CyPass implantation during cataract surgery. Two analysis cohorts were pre-specified based upon medicated baseline IOP. Cohort 1 had initially uncontrolled IOP $\geq 21 \mathrm{mmHg}$, and 40 eyes had a 12-month follow-up visit. At that time, the mean IOP showed $35 \%$ decrease and there was a $49 \%$ reduction in medication usage. The second cohort had initially controlled IOP $<21 \mathrm{mmHg}$. At the 12-month follow-up, 71 eyes were examined and found to have a $75 \%$ reduction in mean medication usage while maintaining mean $\mathrm{IOP}<21 \mathrm{mmHg}$. Fourteen percent of eyes had transient early hypotony, which resolved by 1 month in all cases, without requiring any surgical intervention. A multicenter, singlearm interventional study, using CyPass alone, showed a 35\% reduction in IOP and a $36 \%$ reduction in medication use at 1-year follow-up $(n=65) .{ }^{10}$ Eighty three percent of eyes avoided conventional filtering surgery.

\section{Schlemm's Canal Scaffold}

The Hydrus ${ }^{\mathrm{TM}}$ Microstent (Ivantis, Inc., Irvine, CA, USA) is an $8 \mathrm{~mm}$ long device made of highly elastic biocompatible material called nitinol. It is a crescent-shaped open structure, curved to match the shape of the SC. The idea of this "intracanalicular scaffold" is to maintain SC'S patency over 3 clock hours upon insertion and provide direct aqueous access from the anterior chamber to multiple collector channels. It is currently for investigational use in the United States. The Hydrus II study included 100 patients (50 per treatment arm), who were randomized to have phacoemulsification with Hydrus or phacoemulsification alone. ${ }^{11}$ All patients underwent medication washout prior surgery with baseline diurnal IOP of about $26 \mathrm{mmHg}$ in both arms. At 2-year follow-up the washed-out mean diurnal IOP in the Hydrus plus phacoemulsification group was significantly lower compared with the phacoemulsification alone group, at $16.9 \pm 3.3 \mathrm{mmHg}$ versus $19.2 \pm 4.7 \mathrm{mmHg}$ (50\% versus $28 \%$ reduction), respectively. The proportion of patients using no hypotensive medications was significantly higher at 24 months in the Hydrus plus phacoemulcification group (73\% versus 38\%).

\section{Subconjunctival Implant}

The XEN ${ }^{\circledR}$ Gel Stent (Aquesys Implant) was created by Aquesys Inc (Aliso Viejo, CA, USA). It is made out of a soft collagen-derived gelatin that is known to be non-inflammatory. The goal of the implantation is to create an aqueous humor outflow pathway from the anterior chamber to the subconjunctival space. The implant is $6 \mathrm{~mm}$ long and about the width of a human hair, preloaded in a needle tip injector. Similar to the other implants, it can be performed in conjunction with cataract surgery. It is an investigational device currently undergoing clinical trials.

MIGS technology opens an exciting new chapter in the current glaucoma management. It has the potential to address numerous challenges we face in our daily glaucoma practice, e.g., patient adherence, ocular toxicity, cost of glaucoma medications, etc. MIGS ab interno approach avoids conjunctival manipulation, preserving the more invasive surgery options. Future randomized control studies will hopefully provide us with knowledge about long-term efficacy, ideal patient selection, optimal location for device implantation, and cost-effectiveness data.
1. Saheb $\mathrm{H}$, Ahmed IIK, Micro-invasive glaucoma surgery: current perspectives and future directions, Curr Opin Ophthalmol 2012;23:96-104

2. Francis BA, See RF, Rao NA, et al., Ab interno trabeculectomy: development of a novel device (Trabectome) and surgery for open-angle glaucoma, J Glaucoma, 2006;15:68-73.

3. Ting JL, Damji KF, Stiles MC, Trabectome Study Group. Ab interno trabeculectomy: outcomes in exfoliation versus primary openangle glaucoma, J Cataract Refract Surg, 2012;38:315-323.

4. Mosaed S, Rhee DJ, Filippopoulos T, Trabectome outcomes in adult open-angle glaucoma patients: one year follow-up, Clin surg Ophthalmol, 2010;28:182-186.
5. Francis BA, Trabectome combined with phacoemulsification versus phacoemulsification alone: a prospective, nonrandomized controlled surgical trial, Clin Surg Ophthalmol, 2010;28:1-7

6. Maeda M, Watanabe M, Ichikawa K, Evaluation of trabectome in open-angle glaucoma, J Glaucoma, 2013;22:205-208.

7. Jea SY, Francis BA, Vakili G, et al., Ab interno trabeculectomy versus trabeculectomy for open-angle glaucoma, Ophthalmology, 2012;119:36-42.

8. Malvankar-Mehta MS, Chen YN, Iordanous Y, et al., istent as Solo Procedure for Glaucoma Patients: A Systematic Review and Meta-Analysis, PLoS One, 2015;10:e0128146.
9. Hoeh H, Vold SD, Ahmed IK et al., Initial Clinical Experience With the CyPass Micro-Stent: Safety and Surgical Outcomes of a Novel Supraciliary Microstent, J Glaucoma, 2016;25:106-112.

10. García-Feijoo J, Rau M, Grisanti S, et al., Supraciliary micro-stent implantation for open-angle glaucoma failing topical therapy: 1-year results of a multicenter study, Am I Ophthalmol, 2015;159:1075-1081

11. Pfeiffer N, Garcia-Feijoo J, Martinez-de-la-Casa JM, et al., A Randomized Trial of a Schlemm's Canal Microstent with Phacoemulsification for Reducing Intraocular Pressure in OpenAngle Glaucoma, Ophthalmology, 2015;122:1283-1293. 